\title{
Response to Letter to the Editor: Establishing a threshold to predict risk of cardiovascular disease from the serum triglyceride and high- density lipoprotein concentrations in persons with spinal cord injury
}

\author{
Michael F. La Fountaine ${ }^{1,2,3,4} \cdot$ William A. Bauman ${ }^{1,5,6}$ \\ Received: 31 August 2018 / Accepted: 4 September 2018 / Published online: 26 September 2018 \\ (c) International Spinal Cord Society 2018
}

The authors wish to thank Drs. Stillman and Williams for their Letter to the Editor and for the opportunity to further discuss our recent publication entitled "'Establishing a threshold to predict risk of cardiovascular disease from the serum triglyceride and high-density lipoprotein concentrations in persons with spinal cord injury" (SCI) [1], as well as some aspects of our prior work. Echoing many of the concerns in their Letter to the Editor, we too have struggled with the dearth of empirical evidence on how best to approach cardiovascular disease (CVD) risk-factor stratification, the lack of appropriate guidelines on threshold concentrations for specific lipids at which to intervene, and the scarcity of information on what agent(s) and/or modalities (e.g., diet, exercise) should be used and when, if indicated, in persons with SCI. Our article in this issue of the journal serves to highlight the lack of SCI-specific guidelines on reducing risk factors for CVD, and it also

Michael F. La Fountaine

michael.lafountaine@va.gov

1 Department of Veterans Affairs Rehabilitation Research \& Development Service, National Center for the Medical Consequences of Spinal Cord Injury, James J. Peters Veterans Affairs Medical Center, Bronx, NY, USA

2 Department of Physical Therapy, School of Health and Medical Sciences, Seton Hall University, South Orange, NJ, USA

3 The Institute for Advanced Study of Rehabilitation and Sports Science, School of Health and Medical Sciences, Seton Hall University, South Orange, NJ, USA

4 Departments of Medical Sciences and Neurology, Hackensack Meridian School of Medicine at Seton Hall University, Nutley, NJ, USA

5 Department of Rehabilitation Medicine, Icahn School of Medicine at Mount Sinai, New York, NY, USA

6 Department of Medicine, Icahn School of Medicine at Mount Sinai, New York, NY, USA functions to emphasize the absence of evidence demonstrating the efficacy of CVD risk factor reduction to reduce hard CVD end points (e.g., stroke, myocardial infarction, sudden death due to presumed or proven CVD) in longitudinal cohort studies in persons with SCI. The authors appreciate the multiple challenges to develop research proposals on topics that are of interest to funding agencies, the monumental task to design and execute large-scale clinical trials in persons with SCI, and the perseverance required to generate level 1 evidence that has generalizability across a heterogeneous SCI population, with the outcome aimed to influence how medicine is practiced. Admittedly, due to the multi-factorial etiology for CVD and the possibility of distinct pathophysiological attributes unique to persons with SCI, controversy may exist on the appropriateness of applying guidelines for able-bodied individuals to those with SCI. However, in the absence of clinical trials that address hard CVD end points in the SCI population, clinicians are left to apply accepted practices or research findings that are derived from the general population to support patient care decisions.

The current practice guidelines in the general population favor lowering the low-density lipoprotein cholesterol (LDL-C) concentration as the primary target for CVD risk, and, to a lesser degree, emphasis is placed on increasing the high-density lipoprotein cholesterol (HDL-C) or lowering the triglyceride (TG) concentration. The authors concur with the two main points advanced by Drs. Stillman and Williams concerning our article [1] in this issue of the journal: (1) the clinical benefit of lowering the serum TG concentration to reduce incident CVD events or overall mortality has not been established in persons with SCI, and (2) there is a need for clinical trials in the SCI population to address the efficacy of interventions to reduce hard CVD end points.

The SCI population is limited in number, geographically dispersed, and heterogenous in the usual 
demographic variables, as well as diverse in level, completeness, and duration of lesion. To perform a welldesigned and prospective randomized clinical trial (RCT) on the scale needed to address hard end points for CVD would require thousands of participants who are somewhat uniform in demographic characteristics, as well as somewhat homogeneous for SCI-specific characteristics. Such an undertaking, especially if the participants are relatively young, as is the case for more recently injured men with SCI, usually requires decades of follow-up, such as in the Framingham and Framingham Offspring Heart studies. The question arises as to the feasibility of such an undertaking in the SCI population, and whether the added knowledge would be worth the investment of time, effort, and resources. With these practical considerations in mind, a RCT to address hard CVD end points will not be performed in those with SCI any time soon. Although it is possible to address the efficacy of an intervention to prevent soft CVD endpoints (e.g., carotid intima-medial thickness or coronary artery calcification) in a more limited SCI population, doubts will invariably persist as to the clinical relevance of such RCTs because of lingering questions regarding the accuracy of such findings to predict CVD events. As such, we are left with a fallback position-that is, to rely on epidemiological studies performed in the general population and to draw logical inferences from this work to treat dyslipidemias and other risk factors for CVD in the SCI population, as imperfect and possibly flawed as this approach may be.

With regard to the specific issues raised in our article, controversy exists in the general population as to the benefit of lowering serum TGs, despite several randomized clinical trials that have shown a significant benefit for CVD endpoints, albeit modest, with no benefit to overall mortality rates [2]. However, subgroup analyses of several RCTs have shown that persons who have derived benefit from lowering serum TG levels are those with the lowest initial HDL-C values. Thus, it may be speculated that the greatest benefit of lowering the serum TG concentration could be realized in those with the largest increases in serum HDL-C concentration, especially if the pre-treatment serum HDL-C concentrations are $<40 \mathrm{mg}$ / dl. In our report, when the serum HDL-C concentration was $40 \mathrm{mg} / \mathrm{dl}$, the intersecting serum TG concentration was $115 \mathrm{mg} / \mathrm{dl}$ in those with higher cord lesions $\left(\uparrow \mathrm{T}_{4}\right)$, which was significantly lower than the mean TG value in those with lower cord lesions $\left(\downarrow T_{5}\right)$ and able-bodied controls [1]. In the general population, a serum HDL-C < $40 \mathrm{mg} / \mathrm{dl}$ is recognized as an independent risk factor for CVD, and that the upper limit of normal for a serum TG value is $150 \mathrm{mg} / \mathrm{dl}$. As such, one is left with the quandary of what to do with this new information regarding normal, or even low normal, serum TG concentrations in association with low HDL-C concentrations in persons with higher cord lesions.

In our original work, insulin resistance was present in persons with SCI (all levels of lesion) without, unexpectedly, elevated serum TG levels [3]. Visceral abdominal obesity is well appreciated to be associated with insulin resistance, disorders of carbohydrate handling, dyslipidemia, and hypertension; the serum TG concentration is generally elevated in able-bodied individuals with increased abdominal adiposity. The SCI population has a higher rate of adiposity than the able-bodied population, with a far higher prevalence of abdominal obesity [4]. Thus, the high proportion of those with SCI who have metabolic syndrome or a clustering of independent risk factors for CVD would be anticipated. As such, one would also have anticipated that the serum TG levels should be higher in those with more proximal levels of SCI, but that was not the case, despite similar abdominal adiposity and plasma insulin levels in the groups with higher and lower cord lesions. We speculated that interruption of functional sympathetic innervation to the visceral adipose depot and liver was at least in part responsible for this unexplained observation [5], but appreciate that other factors may still be contributing. As such, the clinician should be aware that a normal or low serum TG concentration does not preclude the possibility that the patient with a higher level of SCI may be obese (by criteria of percent total body adiposity), have sub-clinical insulin resistance and, thereby, be predisposed to CVD.

Our prior works, as well as those of other investigators, have shown that serum HDL-C levels are markedly depressed in persons with SCI, with the lowest values in those with the highest levels of lesion. Universal consensus exists for the clinical benefit of advising lifestyle and/or pharmacologic intervention to reduce adiposity and to promote general health in those who are obese. Furthermore, and in the absence of direct evidence to the contrary, one may hypothesize that by intervening to lower a relatively elevated serum TG level in those with SCI, the serum HDL$\mathrm{C}$ levels may rise to a greater extent than that observed in the able-bodied population, possibly with the beneficial effect of reducing CVD risk and future end-organ disease.

Acknowledgements This work was supported by grant sources from the Veterans Affairs Rehabilitation Research and Development Service (\#B2020-C).

\section{References}

1. La Fountaine MF, Cirnigliaro CM, Hobson JC, Dyson-Hudson TA, Mc Kenna C, Kirshblum SC, et al. Establishing a threshold to predict risk of cardiovascular disease from the serum triglyceride and high-density lipoprotein concentrations in persons with spinal 
cord injury. Spinal Cord. 2018. https://doi.org/10.1038/s41393018-0187-7.

2. Jakob T, Nordmann AJ, Schandelmaier S, Ferreira-Gonzalez I, Briel M. Fibrates for primary prevention of cardiovascular disease events. Cochrane Database Syst Rev. 2016;11:CD009753.

3. Bauman WA, Spungen AM, Zhong YG, Rothstein JL, Petry C, Gordon SK. Depressed serum high density lipoprotein cholesterol levels in veterans with spinal cord injury. Paraplegia. 1992;30: 697-703.
4. Cirnigliaro CM, LaFountaine MF, Dengel DR, Bosch T, EmmonsHindelong R, Asselin $\mathrm{P}$ et al. Visceral adiposity in persons with chronic spinal cord injury determined by dual energy X-ray absorptiometry. Obesity. 2015;23:1811-7.

5. La Fountaine MF, Cirnigliaro CM, Kirshblum SC, McKenna C, Bauman WA. Effect of functional sympathetic nervous system impairment of the liver and abdominal visceral adipose tissue on circulating triglyceride-rich lipoproteins. PLoS ONE. 2017;12: e0173934. 